\title{
A Novel and Low-Cost Method for Measuring Wind Speed and Flow in Pipeline
}

\author{
Xuetang Lei ${ }^{1}$, Huoxi $\mathrm{Xu}^{1}$, Yang Zhao ${ }^{2 *}$ and Jianxin $\mathrm{He}^{3}$ \\ ${ }^{1}$ School of Electronic Information, HuangGang Normal Univercity, \\ Huanggang 438000, China \\ ${ }^{2}$ Department of Electronic and Information Technology, Jiangmen Polytechnic, \\ Jiangmen 529090, China \\ ${ }^{3}$ School of Electronic Information, Huanggang Polytechnic College, \\ Huanggang 438000, China \\ zhaoyang_2015@sina.com \\ Abstract
}

\begin{abstract}
This paper proposes a new method for measuring the wind speed in the pipe, which overcomes the shortcomings of the traditional nechanical instrument with lower accuracy and ultrasonic measurement method with the high cost, it has the advantages of low cost and high precision. This paper introduces the principle of pendulum method to measure wind speed, the wind speed in the pipe is calculated by the space angle based on the spatial geometric relationship. On the other hond, the space angle is obtained by horizontal angle and vertical angle, which are gathered by the cameras placed in the ends of the bracket. Image processing technology is an important part of this method, including the image gray processing, binartzation processing, edge detection and Hough transform steps, at last, the experimental results proved the feasibility of this method.
\end{abstract}

Keywords: wind speed measurement; wind flow; image processing technology; Hough transform

\section{Introduction}

The wind speed measuring system are essential to various domains, such as in the agricultural, meteorological, transport and environmental monitoring domains [1, 4], due to its potential to benefit the investigation and utilization of the wind energy and to improve the production and people's quality of life. The traditional wind speed measuring methods include the mechanical wind measurer and the supersonic wind measuring approach. The mechanical wind speed measuring method is inaccurate and its mechanical parts are prone to be damaged [5].

In [6], ultrasonic wind velocity measurement system was studied based on DSP. The three documents mentioned all adopt the transit-time of the speed difference to measure the wind velocity, and rely on the hardware to improve the accuracy of measurement. In [7], wind velocity measurement and analysis of uncertainty were studied through the ultrasonic sensor and the Kalman filter. Ultrasonic vortex detection system was evaluated in [8]. The supersonic wind speed measuring approach is highly accurate and responsive but it entails high costs, and the temperature variations have impact on the accuracy.

This paper proposes a novel system that measures the wind speed via the simple pendulum. The proposed method first collects the images of the pendulum that moves due to the air flow, and then computes the pendulum's horizontal and vertical deflection angles using the image analysis schemes. Next, the wind speed is computed with the relation between the wind speed and the force applied to the pendulum. The proposed method is inexpensive, easy to implement and highly accurate and thus can be widely 
used for the tubular wind speed measuring system. The proposed method is also capable of computing other parameters (e.g. the flow). The organization of this paper is as follows. The principles of the simple pendulum-based wind speed measuring system are first described. The method for computing the deflection angle via the image processing technique is then presented. And the accuracy of the proposed method is demonstrated by the experiments, thus providing a new approach for measuring the wind speed.

\section{Measuring Principles}

\subsection{Measurement Principle of Wind Speed}

Mutually vertical carriage are erected within the tube, and a pendulum is placed at the intersection of the horizontal and vertical directions along the diagonal, shown as in Figure 1. Assume that the pressure from the air flow is imposed on the pendulum while the air within the tube flows. Suppose that the friction drag that the air applies to the pendulum is negligible (the friction is very slight), then the force applied to the pendulum is mainly from the air flow [9].

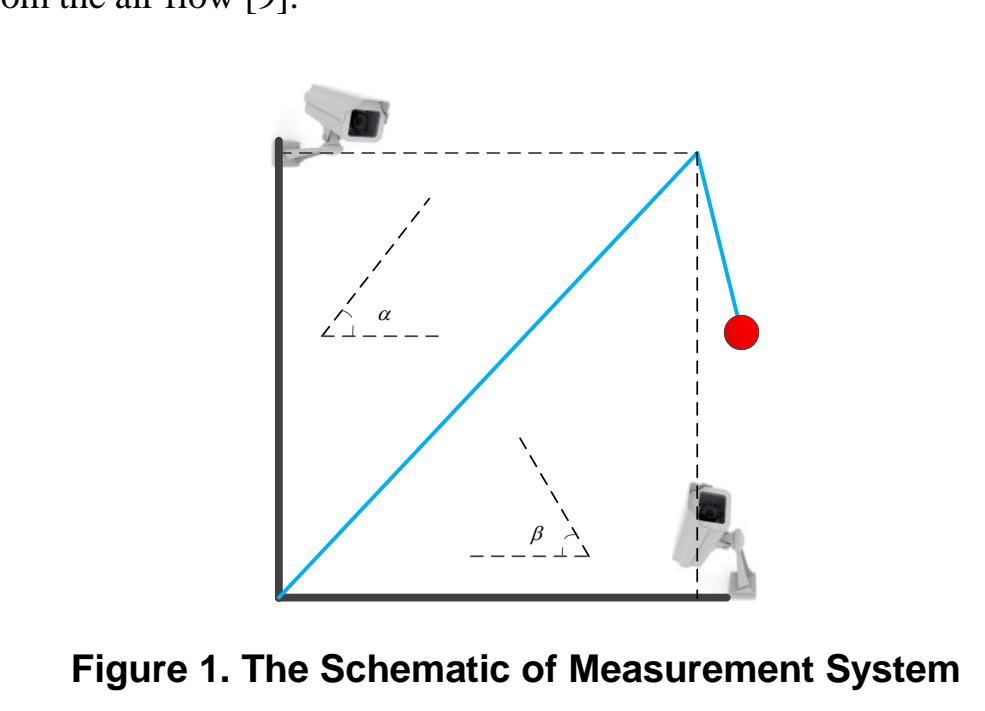

Based on the standard differential pressure formula, the pressure on the pendulum, $\mathrm{P}$, is shown in Equation (0. The higher the wind speed, the greater the pressure on the pendulum.

$$
p=\frac{1}{2} \lambda \rho v^{2}=\frac{F}{S}
$$

Where $\lambda$ denotes the resistance coefficient for the pendulum movement, $\rho$ denotes the air density, $V$ denotes the wind speed, $F$ denotes the force applied to the pendulum, $S$ denotes the force bearing area of the pendulum. Transforming Equation (1), the force bearing formula of the pendulum can be written as:

$$
F=P \mathrm{~S}=\frac{\pi}{8} \lambda \rho R^{2} v^{2}
$$

From the force bearing analysis of the pendulum as shown in Figure 2, the force applied to the pendulum, $F$ can be expressed with Equation (3).

$$
F=m g \tan \theta
$$




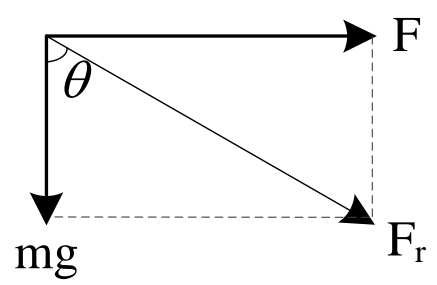

\section{Figure 2. Schematic of Stress Analysis}

Where $m$ denotes the pendulum's quality, $\theta$ denotes the included angle between the net force and the vertical gravity. With Equations (2) and (3), the wind speed within the tube is:

$$
v=\sqrt{\frac{8 m g \tan \theta}{\pi \lambda \rho R^{2}}}
$$

From further analysis of Equation (4), it can be seen that all parametens other than $\theta$ are known. Hence, the wind speed can be computed as rong as $\theta$ is deternined.

\subsection{Measurement Principle of Space Angle $\theta$}

If two cameras are installed along the horizontal॰ and vertical directions, then the deflection angles of the pendulum along the horizontah and vertical directions, $\alpha$ and $\beta$ can be captured. The measurement geometric mode (is) as shown in Figure 3, set $A D=a$, $\mathrm{AB}=\mathrm{b}$, and $\mathrm{AA}_{1}=\mathrm{c}$, and then, we could get:

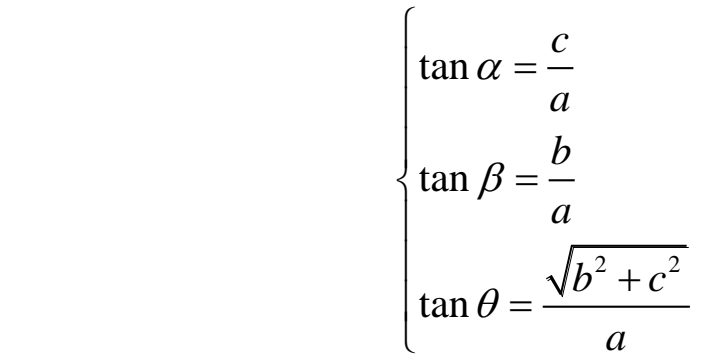

According to the Equation (5), we have $\tan ^{2} \theta=\tan ^{2} \alpha+\tan ^{2} \beta$, from any point in the cross section. So by using the spatial geometric relationship, the direction angle, $\theta$, can be computed with Equation (6).

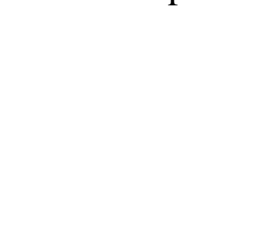

$$
\theta=\arctan \sqrt{\tan ^{2} \alpha+\tan ^{2} \beta}
$$

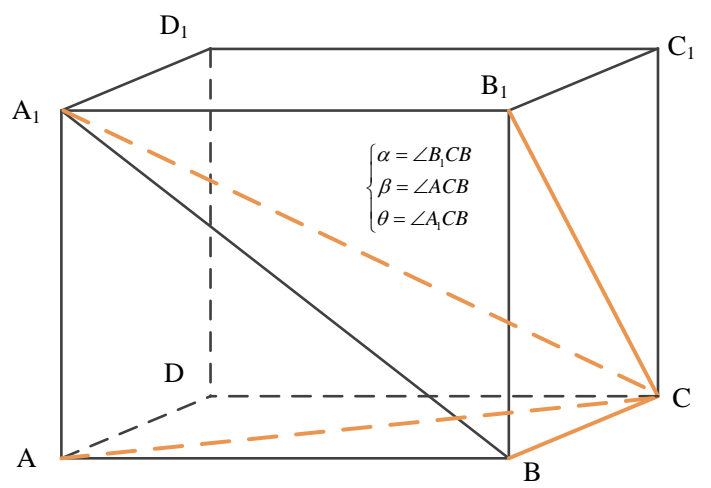

Figure 3. Geometric Module of the Measurement System 


\subsection{Measurement Principle of Flow}

On the assumption that the section for a unit area $\mathrm{d} A$ on the velocity and density is uniform, the volume flow rate through the unit area can be described as in Equation (7):

$$
d q_{v}=\bar{v} d A
$$

In this system, the element is selected as the section center offset $N$ inner radius is $r$, the outer radius of the ring $r+\mathrm{d} r$, ring area is $d A=2 \pi r d r$. Thus, the average velocity in the ring can be calculated by measuring the $M$ points evenly distributed in the ring, which can be described as Equation (8).

$$
\bar{v}=\frac{1}{M} \sum_{1}^{M} v
$$

Furthermore, the average speed of cross section is:

Finally, the mass flow rate is:

$$
\bar{V}=\frac{1}{N} \sum_{1}^{N}\left(\frac{1}{M} \sum_{1}^{M} v\right)
$$

\section{System Hardware Design}

$$
Q_{m}=\rho A \mathrm{~K}
$$

Two fixed skates, L1 and L2, are installed along the diameter direction of the tube's cross section. A guide track, L3, which is the size of the diameter and can move freely, is placed between the two skates so that it can move along the tube. A guide track, L4, which is the size of the radius and can move freely, is placed along the direction vertical to L3. The collecting system can be put of this guide track to move horizontally and vertically. To enable the guide track to move within the tube, a motor is installed on each of L1 and L2, so that L3rean move onthem. A motor can be installed on L3 to enable the collecting system to move in the vertical direction. Two motors can be installed on L4, so that the collecting system can move on them and the guide track can move horizontally.

The controling system execises control via the microcontroller and the step motor, and the block diagram is shown in Figure 2. The four-phase eight-wire step motor is chosen, with a rated voltage of $12 \mathrm{v}$, reduction ratio of $1 / 85$, step angle of $0.9^{\circ}$, and a DC resistance of $130 \Omega$. The control word for driving the step motor is given in Table 1 .

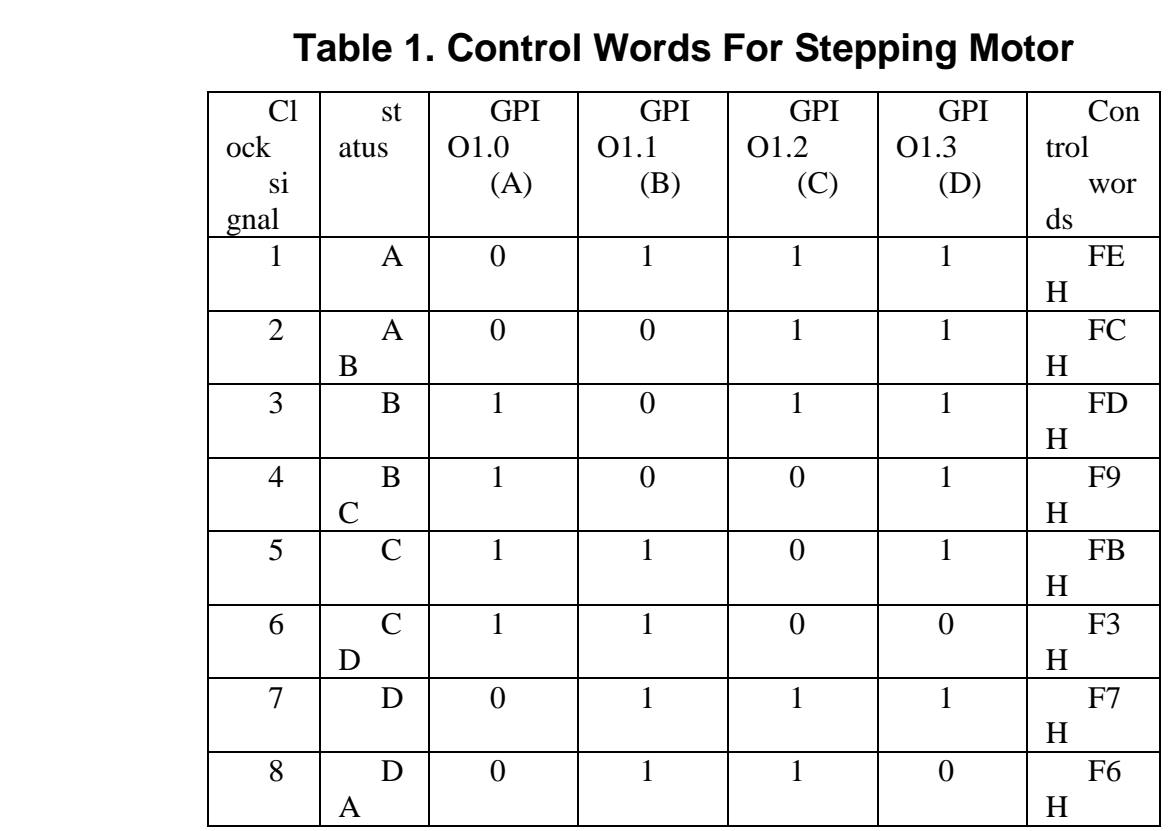




\section{Image Processing Techniques}

The procedures for processing the images in the simple pendulum-based wind speed measuring system are shown in Figure 4, including image collection, grey level transformation, binaryzation, edge detection, and the Hough transform. These steps will be described in detail below.

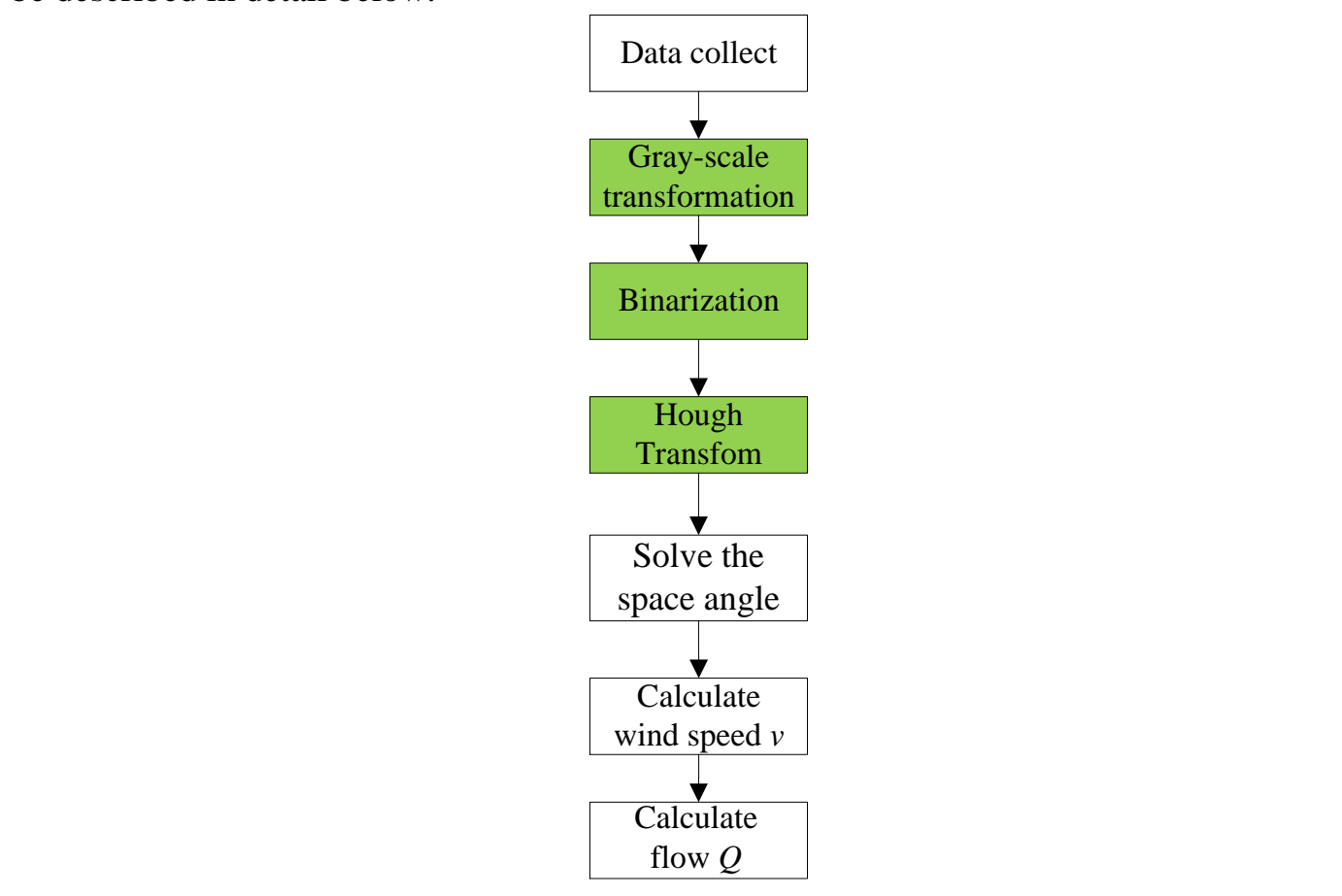

Figure 4. Flow Chart of Figure Process

\subsection{Grey Level Transformation}

The original images captured by the cameras are color images consisting of RGB primary colors. The first step of image processing is to transform the $24 \mathrm{~b}$ pendulum movement color images collected by the cameras into the grey images to facilitate subsequent image processing. The images are transformed into the grey images using the weighted average method.

Let $\mathrm{W}_{\mathrm{g}}$, and $\mathrm{W}_{\mathrm{b}}$ denote the weighted values of $\mathrm{R}, \mathrm{G}$ and $\mathrm{B}$. Generally, our eyes have the greatest sensitivity to green images, followed by the red and then the blue inages. Therefore, we define that $\mathrm{W}_{\mathrm{g}}>\mathrm{W}_{\mathrm{r}}>\mathrm{W}_{\mathrm{b}}$. In this way, we can obtain proper images. In oyn system, the images are processed with the weighted average method. Based on experiments and theoretical derivations, we define the weights of $\mathrm{R}, \mathrm{G}$ and $\mathrm{B}$ as $\mathrm{W}_{\mathrm{r}}=0.3$, $\mathrm{W}_{\mathrm{g}}=0.59$, and $\mathrm{W}_{\mathrm{b}}=0.11$, as shown in Equation (11).

$$
R=G=B=\frac{W_{r} * R+W_{g} * G+W_{b} * B}{3}
$$

\subsection{Binaryzation}

To highlight the features of the pendulum, the binariyzed images can reduce computational loads compared with the grey images, facilitating Boolean operation on the images. More importantly, the binary images can be further processed to obtain the geometrical features (e.g. size, position and shape). There are many binaryzation methods, such as the Bayesian algorithm, Canny algorithm, Otsu algorithm, the fractal dimension- 
based method and the threshold-based method [10]. In this paper, the computation efficient threshold-based method is chosen for binaryzation of grey images.

By setting a threshold $\mathrm{T}$, the data of the image can be divided into two parts. Let $f(\mathrm{x}, \mathrm{y})$ denote the grey image and $\mathrm{g}(\mathrm{x}, \mathrm{y})$ denote the binariyzed image. Then, binaryzation can be done with Equation (12).

\subsection{Edge Detection}

$$
g(x, y)=\left\{\begin{array}{l}
1, f(x, y) \geq \mathrm{T} \\
0, f(x, y)<\mathrm{T}
\end{array}\right.
$$

The commonly used edge detection approaches include the Roberts operator method, the Prewitt operator method, and the Sobel operator method [11]. The Sobel operator method is used in this paper, which is fast and entails slight computational loads. This method needs to compute the grey levels of the chosen point's neighbors, to the left and right, above and below, in a weighted manner, and performs edge detection based on the idea that the weighted grey at the edge point reaches the peak. The $3 \times 3$ template is used as the kernel to perform the convolution sum operation for each pixel in the image, and the computation can be done with Equation (13)

The chosen convolution operators are

$$
\left\{\begin{array}{l}
S_{x}=f(x+1, y-1)+2 f(x+1, y)+f(x+1, y+1) \\
-f(x-1, y-1)-2 f(x y-1, y)-f(x-1, y+1) \\
S_{y}=f(x-1, y+1)+2 f(x, y \notin 1)+f(x+1, y+1) \\
-f(x-1, y-1)-2 f(x, y-1)<f(x+1, y-1)
\end{array}\right.
$$

\subsection{Hough Transform}

Hough transform is one of the basic methods of identifying the geometry from the image in the imageprocessing, the basic principle of Hough transform is to use point and line duality, the orrginal image space, the curve of a given expression through the curve into a parameter space of a point [12-14]. The linear mode commonly used by the Hough transformis given in Equation (14).

$$
\rho=x \cos \theta+y \sin \theta
$$

where $\rho$ denotes the length of the vertical line from the origin to the line, $\theta$ denotes the included angle between the vertical line and the $\mathrm{x}$ axis.

The steps of the Hough transform are given below [15]:

(1) Quantify the discrete parameter space $(\mathrm{k}, \mathrm{b})$ into $\mathrm{m}^{*} \mathrm{n}$ units and construct the accumulator $\mathrm{A}(\mathrm{k}, \mathrm{b})$. Meanwhile, establish the accumulator matrix $O_{\mathrm{m}^{*} \mathrm{n}}$, allocate an accumulator $O(\mathrm{i}, \mathrm{j})$ to each unit in the parameter space, and initialize the accumulator to zero.

(2) Perform the Hough transform on each point in the image space, compute the curve corresponding to the point $(\mathrm{x}, \mathrm{y})$, and increase the accumulator by 1 , i.e. $O_{(i, j)}=O_{(i, j)}+1$.

(3) Determine whether it has proceeded to the final point, find peaks in the accumulators, and the coordinates of the points corresponding to the peaks are the eigenvalues of the parameter space of the line to be detected. 
The straight line equation can be acquired via the Hough transform, and the gradient of the equation is $\tan \alpha$ and $\tan \beta$ of the pendulum in the horizontal and vertical directions.

\section{Experiment and Analysis}

\subsection{Experimental Setup}

The tube has a diameter of $1 \mathrm{~m}$ and a length of $1.2 \mathrm{~m}$. Two fixed guide tracks with a length of $1.2 \mathrm{~m}$ are placed in any direction of the tube's diameter. Between these two guide tracks is placed a 1m-long movable guide track L1, which can slide along the tube. A movable guide track L2 is placed vertically at the center of L1, and the camera (is fixed on L2. In this way, the wind speed at any point within the tube can be measured by moving L1 and L2 horizontally or vertically. A fan with a diameter of about $40 \mathrm{~cm}$ is placed at the entrance to the tube to generate the air flow with stable speed and direction. The pendulum has a quality of $10 \mathrm{~g}$ and a radius of $5 \mathrm{~cm}$. The images of the pendulum at different locations are collected by instructing the two cameras to move horizontally or vertically via the step motor. Then, the wind speed ar this point can be computed. At the standard state, the air density $\rho=1.225 \mathrm{Kg} / \mathrm{m}^{3}$, and the resistance coefficient is usually set to 1 . By substituting these parameters into Equation (4), the instantaneous wind speed within the tube is:

$$
\text { v० }=45.2 \sqrt{\tan \theta}
$$

There are two sets of measuring experiments The first set is to measure the flow field distribution along the center line of the tube, and the second set is to measure the flow field distribution along the cross section of the tube.

\subsection{Measure the Flow Fied Distribution along the Center Line of the Tube}

The supporter moves to the center of the tube via controlling the step motor. Then, the guide track L1 is instructed to move along the tube with a step of $10 \mathrm{~cm}$. There are a total of 13 sampling positions. It stays for $5 \mathrm{~s}$ at each sampling position. At a sampling frequency of $20 \mathrm{~Hz}, 100$ images are collected for each sampling point. The space angle $\theta$ is computed using the image processing techniques. Then, the wind speed at this location can be determined. The 100 data are averaged to reduce measurement errors. The measurement results are given in Figure 5. For the first parts of the distance, the wind speed begins to drep from $23 \mathrm{~m} / \mathrm{s}$ to $20.5 \mathrm{~m} / \mathrm{s}$. After it has passed half the distance of the tube, the wind-speed begins to stabilize.

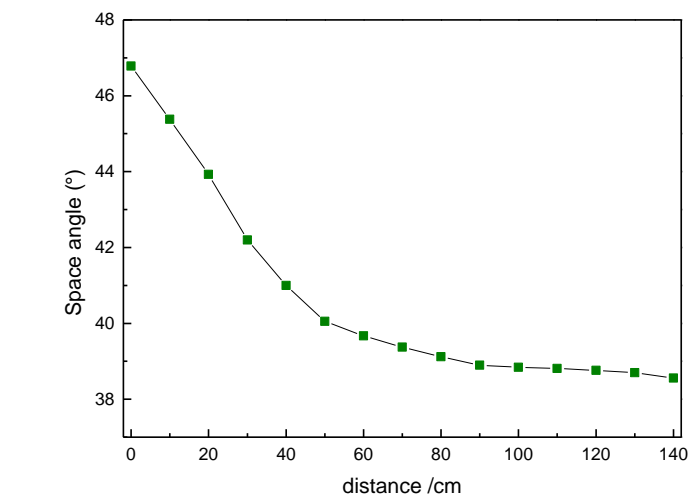

(a)

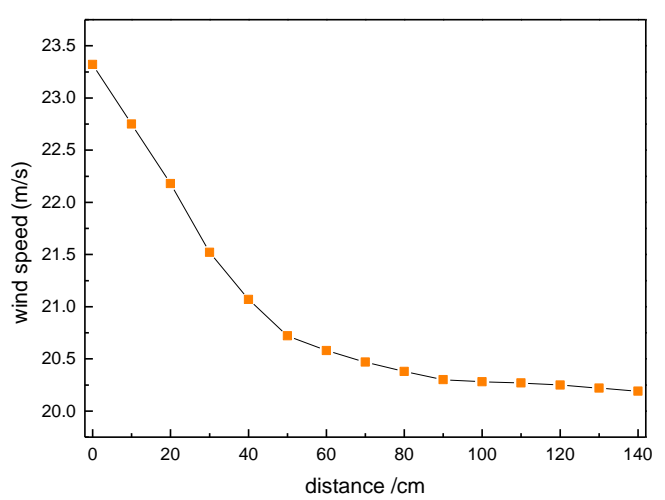

(b) 


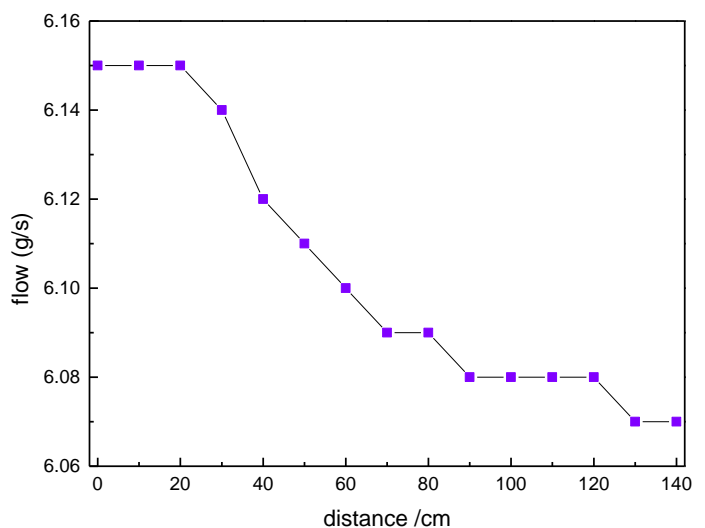

(c)

Figure 5. Distribution of Flow Field along the Pipeline

\subsection{Measure the Flow Field Distribution along The Cross Section Of The Tube}

The supporter is instructed to move to the center of the tribe by controlling the step motor. Then, the guide track L2 is instructed to move from the center of the tube to the wall of the tube with a step of $5 \mathrm{~cm}$. There are total of 10 sampling positions. It stays for $5 \mathrm{~s}$ at each sampling position. At a sampling frequency of $20 \mathrm{~Hz}, 100$ images are collected for each sampling point. The space angle $\theta$ is computed using the image processing techniques. Then, the wind speed af this location can be determined. The 100 data are averaged to reduce measurement errors. Measuring results are shown in Figure 6. From this figure, it can be seen that the wind,speed falls moderately within two thirds of the radius from the center. After this, the speed begins to slump heavily. According to the fluid mechanics theory, the flow field follows the exponential distribution, demonstrating the correctness of the proposed method.

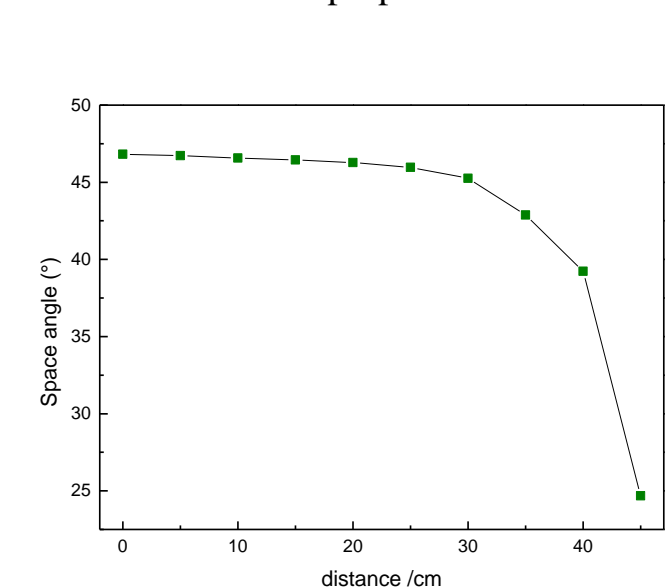

(a)

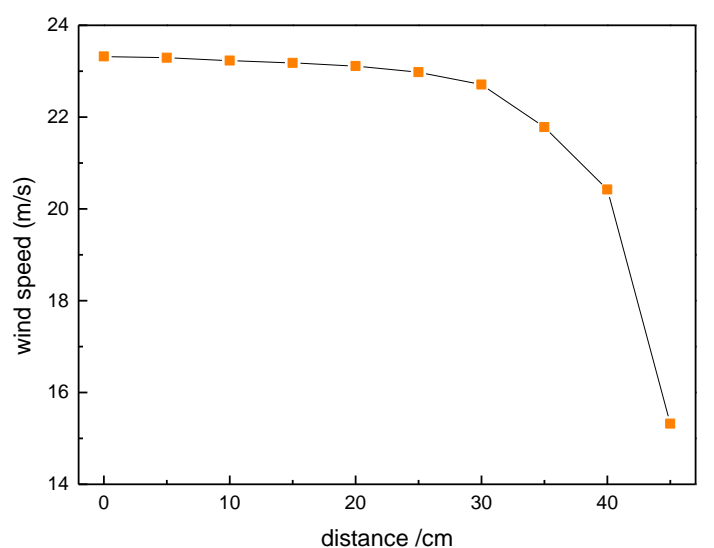

(b) 


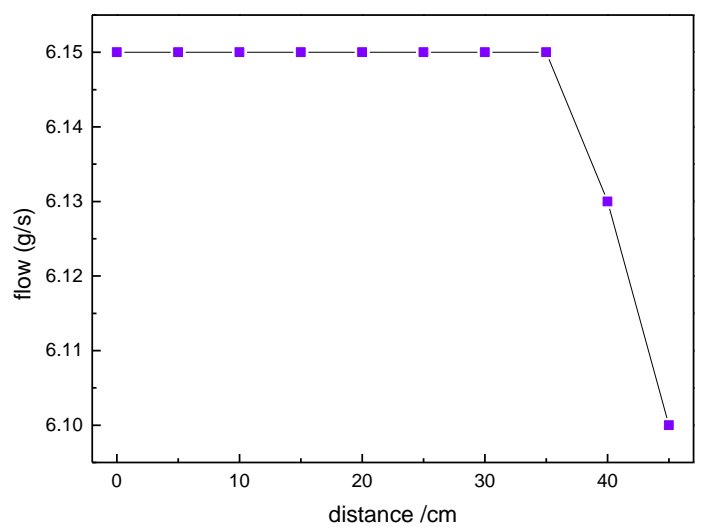

(c)

\section{Figure 6. Distribution of Flow Field along the Cross Section Direction}

\section{Conclusion}

This paper proposes a novel approach that measures the fwind speed using the fluid resistance-based simple pendulum method. We also design an experimental wind speed measuring system. Results show that the proposed method is simple, inexpensive and easy to implement. But accumulated errors may exist daring the computation. Therefore, our future work is to study how to improve the measuing accuracy of the existing system. There are two possible approaches. First, the influence of the environment (especially the air gravity) should be considered (in) the actuat measurement, and the accuracy of the measurement should be compensated according to the actual situation. Second, the Hough algorithm can be improved from the perspective of image processing.

\section{Acknowledgments}

The present research is stpported by the Project of HuangGang Normal Univercity (2014016303; 2015014703).

\section{References}

[1] M. Dupont, C. Celestine and T. Feuillard, "Natural ventilation in a traditional house on a West Indies Island (Guadefoupe) field testing on site and in a wind tunnel", Renewable Energy, vol. 4, no. 3, (1994), pp. $275 \wedge 82$.

[2] H.-J. Krokosźnski, "Efficiency and effectiveness of wind farms-keys to cost optimized operation and maintenance", Renewable Energy, vol. 28, no. 14, (2003), pp. 2165-2178.

[3] A. Lopes, J. Saraiva and M.-J. Alcoforado, "Urban boundary layer wind speed reduction in summer due 10 urban growth and environmental consequences in Lisbon", Environmental Modelling \& Software, vol. 26, no. 2, (2011), pp. 241-243.

[4] S. Palomares, P.-A. Aguera, D.-L. Gonzalez and J.-J. Rosa, "A novel neural network method for wind speed forecasting using exogenous measurements from agriculture stations", Measurement: Journal of the International Measurement Confederation, vol. 55, (2014), pp. 295-304.

[5] C.-Y. Yu, L. Chao and J.-Y. Wang, "Ultrasonic wind velocity measurement based on phase discrimination technique", Telkomnika - Indonesian Journal of Electrical Engineering, vol. 10, no. 6, (2012), pp. 1157-1162.

[6] H.-Q. Tang, W.-Y. Huang, P. Li and Q.-H. Lu, "Ultrasonic wind velocity measurement based on DSP", Journal of Southeast University, vol. 21, no. 1, (2005), pp. 20-23.

[7] C.-E. Espinoza, V. Morales, M. Juan, C. Mauricio and Y. Sebastian, "Wind speed measurement and uncertainty analysis using ultrasonic sensors with Kalman filtering", In: International Instrumentation and Measurement Technology Conference (I2MTC), Austin, USA, (2010), pp.624-628.

[8] D.-A. Pruzan, "Evaluation of an ultrasonic vortex detection system", in: 43rd AIAA, (2005), pp. 55095518. 
[9] L. Tang and W.-B. Shang, "An improved pendulum method for the determination of the center of gravity and inertia tensor for irregular-shaped bodies", Measurement, vol. 44, no. 10, (2011), pp.18491859.

[10] L.-J. Tong, K. Chen, and Y. Zhang, "Document image binarization based on NFCM", in: 2nd IEEE Conference Image and Signal Processing, IEEE Press, (2009) December, pp. 1769- 1773.

[11] E. Cuevas, D. Oliva and D. Zaldivar, "Circle detection using electro- magnetism optimization", Information Sciences, vol. 182, no. 1, (2012), pp. 40-55.

[12] H. Chan, A. Tong, C. Achard and L. Lucat, "Simultaneous segmentation and classification of human actions in video streams using deeply optimized Hough transform", Pattern Recognition, vol. 47, no. 12, (2014), pp. 3807-3818.

[13] J. Dennis, H.-D. Tran and E.-S. Chng, "Overlapping sound event recognition using local spectrogram features and the generalised hough transform", Pattern Recognition Letters, vol. 34, no. 9, (2013), pp. 1085-1093.

[14] R. Hulik, M. Spanel and P. Smrz, "Continuous plane detection in point-cloud data based on 3D Hough Transform", Journal of Visual Communication and Image Representation, vol. 25, no. 1, (2014) pp. 8697.

[15] C. Tu, B.-J. vanWyk, Y. Hamam, K. Djouania and S.-Z. Dub, "Vehicle Position Monitoring Using Hough Transform”, IERI Procedia, vol. 4, (2013), pp. 316-322.

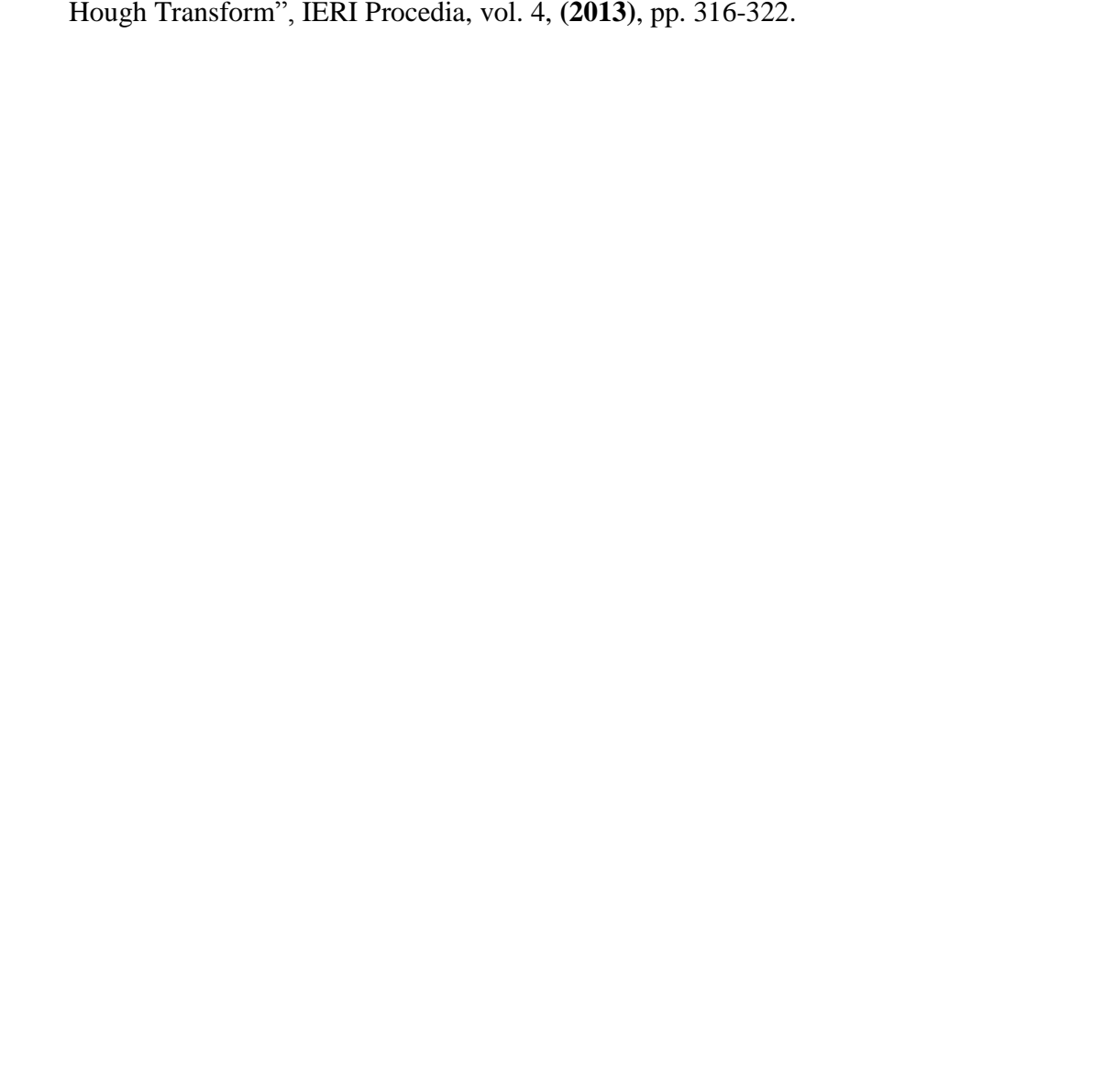

